\title{
INFLUÊNCIA DA TERAPIA NUTRICIONAL NA DOENÇA RENAL CRÔNICA: REVISÃO INTEGRATIVA
}

\author{
Maria Eduarda Oliveira ${ }^{1}$ \\ Tamiris Souza ${ }^{2}$ \\ Ângelo Almeida ${ }^{3}$ \\ Diogenes José Gusmão Coutinho ${ }^{4}$
}

RESUMO: A nutrição exerce um relevante papel na avaliação e no tratamento das doenças renais, uma vez que, a orientação dietética individualizada visa auxiliar no controle e prevenção das possíveis complicações da doença renal crônica (DRC). A DRC é uma enfermidade com características irreversíveis das funções dos rins, normalmente relacionada a diabetes mellitus e hipertensão arterial, que podem avançar para uma fase mais intensa, chamada de doença renal crônica terminal (DRCT). Ao chegar nesse estágio, as funções renais são nulas, deste modo, torna-se necessário o tratamento por meio de hemodiálise (HD) ou diálise peritoneal (DP), com o intuito de substituir as funções renais. Devido ao processo hemodialítico, as perdas de nutrientes são consideradas uma das causas relevantes para a desnutrição, visto que, nesse processo são perdidos vitaminas hidrossolúveis, peptídeos e aminoácidos. Por isso, o estado nutricional desses pacientes é objeto de preocupação e desafio para as equipes multidisciplinares que os atendem. $\mathrm{O}$ presente estudo é de caráter bibliográfico e descritivo, onde foram realizadas buscas nas bases de dados dos últimos seis anos. Assim, os pacientes portadores de doença renal crônica, por serem suscetíveis, necessitam de um acompanhamento constante, para reduzir os agravos da doença, sendo necessário adequar a ingestão de alguns nutrientes, como proteína, cálcio, potássio, fósforo e sódio, visando garantir melhoria no estado nutricional do paciente. Com isso, enfatizamos a importância da terapia nutricional adequada, visando possibilitar melhoria na recuperação e manutenção do estado nutricional.

Palavras-Chave: Doença renal crônica. Hemodiálise. Terapia Nutricional. Dieta. Alimentação Saudável.

ABSTRACT: Nutrition plays a relevant role in the evaluation and treatment of kidney disease, since individualized dietary guidance aims to assist in the control and prevention of possible complications of chronic kidney disease (CKD). CKD is a disease with irreversible characteristics of kidney function, usually related to diabetes mellitus and hypertension, which may progress to a more intense stage, called end-stage renal disease (ESRD). When it reaches this stage, kidney functions are null, so treatment through

\footnotetext{
${ }^{\mathrm{I}}$ Graduanda em nutrição do Centro Universitário Tiradentes, Recife/PE.

${ }^{2}$ Graduanda em nutrição do Centro Universitário Tiradentes, Recife/PE

3 Mestre professor orientador do Curso de Nutrição, do Centro Universitário Tiradentes

Pernambuco.

${ }^{4}$ Professor Doutor em Biologia pela UFPE. E- mail: gusmao.diogenes@gmail.com.
} 
hemodialysis (HD) or peritoneal dialysis (PD) becomes necessary in order to replace kidney functions. Due to the hemodialysis process, the loss of nutrients is considered one of the relevant causes for malnutrition, since in this process water-soluble vitamins, peptides and amino acids are lost. Therefore, the nutritional status of these patients is a matter of concern and a challenge for the multidisciplinary teams that care for them. The present study has a bibliographic and descriptive character, where searches were made in databases over the last six years. Thus, patients with chronic kidney disease, for being susceptible, need constant monitoring to reduce the aggravations of the disease, and it is necessary to adjust the intake of some nutrients such as protein, calcium, potassium, phosphorus and sodium, aiming to ensure improvement in the patient's nutritional status. Thus, we emphasize the importance of adequate nutritional therapy, aiming to enable improvement in the recovery and maintenance of the nutritional status.

Keywords: Chronic kidney disease. Hemodialysis. Nutritional Therapy. Diet. Healthy Eating.

\section{INTRODUÇÃO}

A Doença renal crônica (DRC), na atualidade, é definida como um termo análogo de desordens heterogêneas que atingem a função e estrutura dos rins existentes, por um período superior a 3 meses e que traz comprometimentos à saúde. Essa definição é fundamentada em três componentes: o anatômico ou estrutural, que são considerados marcadores de dano renal, o funcional, que está associado a taxa de filtração glomerular (TGF) e o temporal (CUPPARI, 2019).

Segundo dados do Censo Brasileiro de Diálise, com análise de dados da década 2009-2019, a estimativa de pacientes com doença renal crônica dialítica ultrapassava os 130.00o, com registro no aumento da prevalência e da incidência de pacientes em tratamento dialítico (NEVES, 2018). Dados nacionais revelam que as principais causas de DRC são: diabete mellitus, hipertensão arterial, doença renal policística e as doenças glomerulares, estas causas podem levar pacientes para alguma modalidade dialítica (MARTINS, 2016).

A DRC pode ser definida por estádios, de acordo com os resultados da taxa de filtração glomerular e na excreção de albumina na urina, a partir desses serão avaliados a redução e comprometimento da função renal (LIMA, 2021). No decorrer da doença é frequente o aparecimento de complicações próprias devido a perda da função, como anemia, desnutrição, perda óssea, acidose metabólica e episódios de complicações cardiovasculares (PAZ, 2016). 
Em resposta a uma diminuição da taxa de filtração glomerular, o rim é induzido a uma sequência de adequações, visando a prevenção da doença, onde nota-se uma melhoria na TFG a curto prazo, porém, em longo prazo resulta na perda rápida de néfrons, consequentemente a uma insuficiência renal crônica. Em resposta a redução no números de néfrons, surge a hiperplasia renal, resultando no aumento da TFG e na perfusão renal. Com isso, ocorre a perda da seletividade, consequentemente impedindo a passagem de macromoléculas para a urina (TELLES, 2015).

Com a progressão da DRC para a fase terminal, surge a necessidade da terapia dialítica, onde a taxa de filtração glomerular é $<15 \mathrm{~mL} / \mathrm{min} / \mathrm{m}^{2}$. Estado este, em que os rins são incapazes de manter a filtração sanguínea, o volume, a composição iônica, a síntese e degradação de enzimas e dos hormônios (AQUINO, 2016). É nesse momento que surgem os tratamentos, um deles é a hemodiálise (HD), processo onde um equipamento (dialisador) filtra e limpa o sangue, desempenhando a função que os rins estão incapazes de realizar. Nesse tratamento é liberado o acúmulo de soluto, eletrólitos e água do corpo do paciente, através de um cateter comumente localizado na veia subclávia ou por meio de uma fístula arteriovenosa. A hemodiálise também é responsável em regular a pressão arterial, e auxilia mantendo o equilíbrio de substâncias como a creatinina, potássio, sódio e ureia (RODRIGUES, 2017).

Estudos recentes apontam que a alta carga de ácido dietético pode diminuir a função renal e que a acidose metabólica contribui para a progressão da doença renal crônica. As dietas alcalinas, baseadas em frutas e verduras, têm o potencial de neutralizar a produção de ácidos decorrente do consumo excessivo dos alimentos acidificantes, como as carnes e os queijos (SILVA, 2020). Um padrão alimentar mais sadio está correlacionado a uma menor probabilidade de uma doença renal em estágio final, isto implica em melhores taxas de sobrevida nos paciente com doença renal crônica, auxiliando na diminuição da inflamação sistêmica (LUDVIG, 2019).

Vale ressaltar que a importância da terapia dietética, diminui a progressão da doença para os estágios mais graves, tendo como finalidade prevenir ou amenizar os sintomas, mantendo o paciente em um bom estado nutricional. O fator dietético está associado ao agravamento das condições metabólicas, uma correta intervenção terapêutica tem como principal objetivo minimizar alterações metabólicas podendo tornar o paciente 
menos assintomático, assim possibilitando melhorias na qualidade de vida. É primordial e indispensável que este paciente siga as orientações nutricionais, levando em consideração principalmente o consumo de potássio, fósforo e da proteína, pois, estes estão ligados aos principais agravos referente à patologia existente (CASTRO, 2018).

Devido às dificuldades enfrentadas pelos pacientes renais crônicos, o presente trabalho teve como principal objetivo revisar, através de artigos científicos, a importância da terapia nutricional em pacientes renais crônicos, visando manter um bom estado

nutricional, prevenindo ou minimizando sintomas, consequentemente promovendo uma melhor qualidade de vida desses pacientes.

\section{METODOLOGIA}

Caracterizado como um estudo bibliográfico, de caráter descritivo, com análise de referências obtidas nas seguintes bases de dados: as bases essenciais Literature Analysisand, Retrieval System online/PubMed (Medline) e Literatura Científica e Técnica da América Latina e Caribe/Biblioteca Virtual em Saúde (LILACS) a base opcional Scientific Eletronic Libracy Online (Scielo), buscadores na web e literatura cinzenta (busca manual). Para identificar todas as publicações relevantes, foram realizadas buscas nas bases de dados quanto aos últimos seis anos, de 2015 a 2021.

A estratégia de busca foi definida por descritores cadastrados nos Descritores em Ciências da Saúde (DECs), com o unitermo "protocolo" em combinação com termos relativos ao estado nutricional do paciente renal crônico (DRC). Os critérios de inclusão foram definidos previamente: artigos redigidos nos idiomas português, inglês e espanhol; estudos realizados em DRC e manuscritos publicados nos últimos cinco anos. Os critérios de exclusão de artigos compreenderam: artigos de revisão não disponíveis na versão completa, comunicações breves e artigos, artigos indisponíveis quando solicitado aos autores; que não se referirem aos critérios definidos para inclusão. A seleção dos estudos foi realizada identificando o total de referências da busca de dados nas bases eletrônicas e na busca manual, excluindo as duplicadas, e em sequência, uma triagem por meio da leitura do título e resumo.

As referências selecionadas foram analisadas por completo, nas quais, foi feita a extração dos dados (autor, ano de publicação, amostra do estudo, objetivo, uso de protocolo 
nutricional). Os manuscritos foram avaliados quanto à principal questão da pesquisa: intervenção da terapia nutricional. Todos os artigos que se enquadram com o desenho do estudo, foram incluídos na avaliação.

\section{OS RINS}

Localizado na cavidade abdominal posterior, possuindo cerca de 12 centímetros de comprimento, 2,5 centímetros de espessura e 5 centímetros de largura, sendo protegido por uma camada de músculos, localizando-se um de cada lado da coluna vertebral. Devido a localização do fígado, o rim direito fica um pouco mais baixo que o esquerdo. Considerados órgãos cruciais para manter uma boa homeostase do corpo humano, exercem uma função vital e são encarregados pela filtração sanguínea, pela regulação do volume de líquidos e eliminação do excesso de água, sais e substâncias indesejadas. Além disso, produzem hormônios e reabsorvem nutrientes essenciais (MESTRINHO, 2019).

Cada rim possui cerca de um milhão de néfrons, considerados unidades funcionais. Os néfrons consistem em um glomérulo que contém as artérias eferentes e aferentes, a cápsula de Bowman, o túbulo contorcido proximal e distal, a alça de Henle e os dutos coletores. Por ter como principal função a filtração das toxinas do corpo humano e a excreção através da urina, ocorrendo algum declínio na função renal, este órgão começa a apresentar dificuldade de regulação e excreção das substâncias, acarretando acúmulos na corrente sanguínea. Por consequência, haverá redução na taxa de filtração glomerular, quanto menor essa medida, maiores serão os danos na função renal que serão percebidos pelos primeiros sinais e sintomas. (RIBEIRO, 2020). Consequentemente, com o avanço da doença para os estádios 4 e 5 , serão indicadas as terapias renais substitutivas (TRS), dentre elas, estão os tratamentos dialíticos: hemodiálise (HD), diálise peritoneal (DP) ou o transplante renal. (BORGES, 2019).

\section{DOENÇA RENAL CRÔNICA (DRC)}

A doença renal crônica (DRC) é uma enfermidade com características irreversíveis das funções dos rins, normalmente relacionada a diabetes mellitus e hipertensão arterial, a DRC é classificada em estágios que variam de acordo com a taxa de filtração glomerular e na excreção de albumina na urina, serão avaliados a redução e comprometimento da função 
renal. O estágio $\mathrm{I}$, considerado normal ou elevado com taxa $\geq 90 \mathrm{TFG}\left(\mathrm{mL} / \mathrm{min} / \mathrm{r}, 73 \mathrm{~m}^{2}\right)$; estádio 2, nota-se uma redução leve; estádio $3 \mathrm{~A}$, redução leve a moderada; estádio ${ }_{3} \mathrm{~B}$, redução moderada a grave; estádio 4, redução grave; estádio 5, falência renal (LIMA, 202I). Ao chegar no estágio 5, já é classificada como doença renal crônica terminal (DRCT). Quando chega nesse estágio, as funções renais são nulas, deste modo, torna-se necessário o tratamento por meio de hemodiálise (HD) ou diálise peritoneal (DP), com o intuito de substituir as funções renais (FUKUCHIMA, 20I6).

Destaca-se que, as doenças crônicas atingem um grupo de patologia que gera alterações no estilo de vida. Teoricamente, tais doenças têm uma história prolongada, onde os sintomas aparecem de forma gradual, apresentando um período longo, assintomático, seguido de vários fatores de risco, que sofrem interferência do ambiente. $\mathrm{O}$ paciente com DRC não tem o certo conhecimento de fato, onde seu diagnóstico tardio pode dificultar sua cura, permitindo o estado crônico da doença. A descoberta e o tratamento precoce em estágios iniciais ajudam a prevenir a conclusão maléfica, decorrente da morbidade relacionada à nefropatia. Com o aumento dos estágios da DRC, o paciente pode apresentar dificuldades físicas, onde impedem a sua autonomia, exigindo ajuda e dedicação de familiares nas diversas tarefas. Sobretudo, a população com DRC, também tem aumentado proporcionalmente ao envelhecimento da população em geral e o aumento de pacientes com hipertensão arterial e diabetes mellitus, que a princípio, são os principais agravantes dessa patologia (RIBEIRO, 2020).

\section{I DOENÇAS ASSOCIADAS}

A hipertensão arterial sistêmica (HAS) atinge cerca de $30 \%$ da população no mundo, onde os alimentos industrializados são um dos principais motivos para o agravamento da doença e, consequentemente, para o avanço do estágio terminal da DRC. A doença renal crônica pode agravar com complicações referentes à HAS, sendo um preditor livre de doença cardiovascular (DCV). A despesa geral da DRC é ponderada a I,3\% do orçamento de saúde, dos quais $13 \%$ são referentes ao excesso de infarto agudo do miocárdio e acidentes vasculares cerebrais. A possibilidade do perigo aumenta na medida em que a taxa de filtração glomerular estimada (TFCe) vai caindo e os níveis de albuminúria vão aumentando. Nesse caso, um acompanhamento da pressão arterial (PA) é 
de extrema importância, em especial para pacientes com DRC para que evite complicações e a progressão da doença (STOMPÓR T, 2020).

Uma das patologias mais frequentes a se agravar em pacientes com DRC é a anemia, acarretando a falta de nutrientes, principalmente o ferro e a vitamina $\mathrm{B}_{12}$, devido a má absorção no sistema gastrointestinal. Na relação entre a anemia e a DRC ocorre uma perda na ação da eritropoietina que é gerada pelo estado inflamatório acelerado da doença. A eritropoietina é identificada por um hormônio que ajuda na proliferação e diferenciação das células hematopoiéticas (NUHU, F., \& BHANDARI, 2018).

A anemia pode trazer diversas consequências para o paciente portador de DRC, tendo em vista os sinais e sintomas como, fadiga, perda da capacidade cognitiva, dispneia, ainda tendo uma grande chance ao aumento de doenças cardiovasculares, podendo levar o paciente a óbito (HONDA, H. et al., 2019).

$\mathrm{Na}$ DRC, a anemia pode ser localizada no momento do diagnóstico, sendo a principal causa a redução da capacidade dos rins na produção de eritropoietina (EPO). Porém, a deficiência de ferro e outros fatores associados a ação inflamatória crônica da DRC, consegue contribuir no desenvolvimento dessa anemia (AKIZAWA, T., OKUMURA, et al, 2018).

\subsection{TERAPIA RENAL SUBSTITUTIVA}

A diálise é uma terapia utilizada para remover os acúmulos de solutos urêmicos e o excesso de água, através dela permite-se o restabelecimento do equilíbrio eletrolítico e ácido básico do paciente. De acordo com a Sociedade Brasileira de Nefrologia, esse tratamento é efetuado através da ligação do paciente a um equipamento dialisador, que por sua vez possui a tarefa de realizar parte da função renal. $\mathrm{Na}$ hemodiálise (HD) ou na diálise peritoneal (DP) o plasma urêmico é posto em contato com a solução de diálise, separados unicamente por uma membrana permeável, que na HD é uma membrana artificial, já na DP é a própria membrana do peritônio (CUPPARI,2019).

Dentre os métodos de terapia dialítica, a hemodiálise (HD) é mais utilizada. Para realização de uma sessão, o paciente precisa ter algum tipo de acesso vascular, dentre eles: a fístula arteriovenosa, o enxerto $\mathrm{AV}$ ou o cateter venoso. Quando este paciente é conectado à máquina, dá-se início a sessão, processo onde o sangue passa por dentro de um 
tubo composto de uma membrana de diálise, ocorrendo a difusão entre o sangue e o líquido de diálise circundante, removendo o excesso de líquidos, realizando a depuração do sangue, retirando a ureia, o potássio, o fósforo, o ácido úrico e as moléculas. Esse processo pode durar cerca de 2 a 5 horas e as sessões podem ser de duas a três vezes por semana (TELLES, 2015).

Considerada crucial, as sessões de hemodiálise evitam que os pacientes com DRC vá a óbito, mesmo sabendo que as sessões não compensam as perdas das atividades endócrinas ou metabólicas realizada pelos rins, o paciente precisa saber da extrema importância desse tratamento para prolongar o seu tempo de vida (RIBEIRO, 2020). Sabendo-se que, com a redução ou a perda da função renal, surgem inúmeros distúrbios hormonais, hidroeletrolítico e metabólicos que favorecem indireta ou diretamente para a evolução de uma situação nutricional instável, definidos pela depleção de reservas de gorduras e proteína, principalmente de tecido muscular (CUPPARI, 2019).

\section{PROTEÍNA}

A restrição do aporte protéico tem sido bastante utilizada na prescrição dietética dos pacientes com DRC. Estudos mostram que a ingestão aguda de proteína aumenta o fluxo plasmático renal, a taxa de filtração glomerular, a pressão intraglomerular e influência na hemodinâmica renal, e a sua diminuição pode auxiliar no controle da DRC, favorecendo melhoria nos níveis de albuminúria e no controle da uremia (PEREIRA, 2020).

Devido a escassez de estudos clínicos, a recomendação ideal de proteínas para pacientes em terapia renal substitutiva (HD ou DP), ainda é dubitável. Com base nos escassos estudos, estima-se que para paciente em diálise, o teor ( 1,1 e I,2 g/ $\mathrm{kg} / \mathrm{dia}$ ) de proteínas é essencial, devido ao maior grau de catabolismo protéico e a perdas de aminoácidos durante as sessões de hemodiálise. (CUPPARI, 2019). Por este motivo, indica-se o fornecimento de refeições saudáveis, antes, durante e após cada sessão de diálise, com propósito de atenuar o catabolismo protéico (PEREIRA, 2020).

Uma alimentação inapropriada pode ocasionar o aceleramento da sarcopenia, a perda progressiva de massa muscular pode estar associada a risco de infecções e fraturas (AQUINO, 2016). A ingestão de proteína deve ser acompanhada rigorosamente, visto que, 
no processo de diálise ocorrem perdas significativas dessa macromolécula, podendo levar a diversas complicações no estado clínico do paciente, dietas com ingestão protéica inferior a $\mathrm{Ig} / \mathrm{kg} /$ dia, pode está associado à desnutrição e ao aumento das taxas de mortalidade desses pacientes (PLÁCIDO, 202I).

\section{I POTÁSSIO}

O consumo do potássio merece atenção na doença renal crônica, especialmente nas fases mais avançadas onde a hipercalemia prevalece. Com a restrição da excreção renal, alguns fatores favorecem para o ganho do potássio sérico, sendo eles, a acidose metabólica, constipação intestinal, diabetes mellitus, uso de inibidores RAAS, beta-bloqueadores e a ingestão de alimentos com alto teor de potássio. Sendo assim, todos esses fatores devem ser examinados para evitar e tratar a hipercalemia (CUPPARI, 2019).

O potássio pode ser encontrado em laticínios e carnes, que são alimentos de origem animal e nos alimentos de origem vegetal, como frutas, nozes e legumes. Vale ressaltar que os vegetais não necessitam serem excluídos da refeição, o que deve ser feito é a adequação

da dieta visando ofertar alimentos que possuem um menor teor de potássio, visto que são fontes de minerais, vitaminas e compostos bioativos. No momento da preparação das refeições, é indicado cozinhar alimentos de origem vegetal em bastante água, após fervura, descartar a água utilizada no cozimento, esse procedimento auxilia na diminuição do teor de potássio desses alimentos. (PEREIRA, 2020).

\subsection{FÓSFORO E CÁLCIO}

Encontrado em quantidades consideráveis nos alimentos fontes de proteínas vegetais (oleaginosas, leguminosas e grãos de cereais) e animais (laticínios, carne bovina, aves, peixe, ovos) (ROSS, 2016), o consumo de fósforo deve ser restrito em pacientes com DRC, uma vez que, é frequente o desenvolvimento de hiperfosfatemia, decorrente da baixa eficácia da sua remoção durante o processo dialítico (CUPPARI, 2019).

Por isso, é recomendado restringir o consumo de alimentos protéicos da dieta do paciente com DRC, o profissional deve estar atento e priorizar os alimentos com baixa razão fósforo-proteína. Também é indispensável a restrição de alimentos que não interferem na ingestão protéica, como as vísceras, os miúdos, as oleaginosas e os alimentos 
que contêm uma quantidade considerável de fósforo devido aos conservantes utilizados (AQUINO, 2016).

A homeostase do fósforo fica comprometida, à medida que a função renal diminui. Com isso, o controle inapropriado do fósforo também pode estar ligado ao surgimento do hiperparatireoidismo, por isso, métodos devem ser adotados para diminuição e controle nos níveis de fósforo. Além da orientação nutricional, é primordial uma diálise adequada e a utilização de quelantes, de acordo com a ingestão de fósforo nas refeições (SILVA, 2016).

Os quelantes de fósforo atuam ligando-se em uma porção do alimento no trato gastrointestinal, criando um composto insolúvel que não pode ser absorvido, consequentemente será eliminado através das fezes. Por isso, a importância dos pacientes consumirem os quelantes junto às refeições que contenham esses alimentos com alto teor de fósforo (CUPPARI, 2019).

Com o acúmulo do fósforo e os níveis de vitamina $\mathrm{D}$ diminuídos, o organismo acaba não utilizando o cálcio de maneira adequada. Como consequência, busca corrigir retirando o cálcio dos ossos direcionando para o sangue. Quando ocorre a diminuição da função renal, tendem a ocorrer mudanças na dinâmica do cálcio, ocasionando a hipocalcemia (deficiência de cálcio no sangue), resultando na debilidade nos dentes e ossos, câimbras, dificuldades coagulação e redução dos batimentos cardíacos. Visando a prevenção deste fato, é fundamental equilibrar os níveis de fósforo e cálcio na dieta do paciente com DRC (TELLES, 2015).

\subsection{SÓDIO E INGESTÃO HÍDRICA}

A restrição de sódio é recomendada para controle da pressão arterial e para o controle da ingestão de líquidos, visando a redução do ganho de peso interdialítico. É recomendado de 5 a $6 \mathrm{~g}$ de sal (cloreto de sódio) por dia, ou seja,

2.000 a $2.300 \mathrm{mg} / \mathrm{dia}$ de sódio, metade da quantidade de sódio que é ingerido pelos brasileiros, justificando a dificuldade dos pacientes a seguirem essa orientação (CUPPARI, 2019). Segundo os pacientes portadores de DRC, a maior barreira para adesão à dieta prescrita seria o sabor dos alimentos por conta da restrição do sal. Para eles o sal utilizado na maioria das preparações é um ingrediente importante no sabor final dos alimentos (LAMBERTI, 2017). Desta forma, visando a redução no consumo, os profissionais devem 
orientar seus pacientes a realizarem trocas de temperos industrializados por temperos naturais, ressaltando também que eles não devem consumir produtos em conservas, embutidos e enlatados por terem um alto teor de sódio (CUPPARI, 2019).

Com a progressão da doença renal, ocorre a diminuição da capacidade em excretar os líquidos, ocasionando dificuldade em manter o débito urinário habitual. Pacientes que estão em hemodiálise (HD) a ingestão hídrica baseia-se na capacidade de excreção de água pela urina e suor. O melhor indicador para adequação da ingestão hídrica é o ganho de peso interdialítico, porém de modo geral a recomendação é de 500 a $1.000 \mathrm{~mL} /$ dia acrescentados ao volume de diurese das 24 horas. Os pacientes que realizam a diálise peritoneal (DP), costumam se preocupar menos com a ingestão hídrica, visto que as sessões são diárias. Lembrando que todos os alimentos que contém líquidos devem ser considerados nos cálculos, como por exemplo a melancia, sopas, café e gelatinas. Para aliviar a sede, profissionais orientam que seus pacientes façam consumo de pequenas quantidades de água gelada ou até mesmo chupem pequenas pedras de gelo (AQUINO, 2016).

\section{TERAPIA NUTRICIONAL NA DOENÇA RENAL CRÔNICA}

A nutrição executa um relevante papel na avaliação e no tratamento das doenças renais, uma vez que, a orientação dietética individualizada visa auxiliar no controle e prevenção das possíveis complicações da DRC. Devido ao processo hemodialítico, as perdas de nutrientes são consideradas uma das causas relevantes para a desnutrição, visto que, nesse processo são perdidos vitaminas hidrossolúveis, peptídeos e aminoácidos. Por isso, o estado nutricional desses pacientes é objeto de preocupação e desafio para as equipes multidisciplinares que os atendem (PERUSSO, 2019).

A dieta é um fator essencial e determinante podendo trazer vários benefícios para os pacientes com DRC, visando assim a manutenção do equilíbrio ácido-básico. Uma alimentação rica em proteína animal tende a gerar um aumento na produção de ácido, já a alimentação rica em vegetais e frutas geram bicarbonato e reduzem a carga líquida de ácidos na dieta (BANERJEE, 2016).

Sabendo que o rim é um órgão denominado como filtrador, quanto menor a carga gerada pelo metabolismo, menor será o trabalho renal. Por este motivo, o profissional deve 
estar atento ao estágio da doença para assim adequar a dieta, visando controlar ou minimizar os sintomas da DRC (CASTRO, 2018).

O planejamento dietético desse paciente irá depender da condição clínica atual em que ele se encontra. Por isso, deve ser observado o principal motivo desse paciente se encontrar no seu estado atual, ou o que levou ao acometimento dessa doença de base, e se há outras patologias associadas a ela, como por exemplo a hipertensão arterial e a diabetes mellitus (PLÁCIDO, 2021).

\section{RESULTADO E DISCUSSÕES}

Tabela I. Relação dos estudos realizados sobre a influência da terapia nutricional na doença renal crônica (2016 - 202I).

\begin{tabular}{|c|c|c|c|}
\hline AUTOR / ANO / TÍTULO & UTOR (es) & $\begin{array}{c}\text { ANO DE } \\
\text { UBLICAÇÃO }\end{array}$ & OBJETIVOS \\
\hline $\begin{array}{l}\text { Terapia nutricional em } \\
\text { pacientes com Doença Renal } \\
\text { Crônica: Revisãonarrativa }\end{array}$ & PLÁCIDO ES, et al & 2021 & $\begin{array}{l}\text { Revisar a importância da terapia nutricional } \\
\text { nos pacientes portadores da doença renal } \\
\text { ônica, enfatizando sobre anecessidade de um } \\
\text { companhamento doestado nutricional desses } \\
\text { pacientes. }\end{array}$ \\
\hline $\begin{array}{l}\text { Diet in Chronic Kidney } \\
\text { isease: an integratedapproach } \\
\text { to nutritional } \\
\text { therapy. }\end{array}$ & PEREIRA RA, et al & 2020 & $\begin{array}{l}\text { Enfatizar os benefícios da adesão da dieta } \\
\text { limentar associado a melhores resultadospara } \\
\text { a prevenção e tratamento da doença renal } \\
\text { crônica (DRC). }\end{array}$ \\
\hline $\begin{array}{c}\text { Alimentação e hábitos devida na } \\
\text { doença renal crônica. }\end{array}$ & $\begin{array}{l}\text { PERUSSO GKF, et } \\
\text { al }\end{array}$ & 2019 & $\begin{array}{c}\text { Relacionar a dieta e o estilo de vida dos } \\
\text { pacientes com DRC. }\end{array}$ \\
\hline $\begin{array}{l}\text { An integrative review of the } \\
\text { ethodology and findings regarding } \\
\text { dietary adherence in end stage } \\
\text { kidney disease. }\end{array}$ & LAMBERT K, et al & 2017 & $\begin{array}{l}\text { elatar os métodos utilizados para determinar } \\
\text { adesão à dieta e associar osfatores que podem } \\
\text { impactar diretamente aessa adesão. }\end{array}$ \\
\hline $\begin{array}{l}\text { Dietary Patterns and CKD } \\
\text { Progression. }\end{array}$ & BANERJEE $T$, et al & 2016 & $\begin{array}{c}\text { Analisar o papel da dieta, enfatizando os } \\
\text { padrões alimentares e progressão da doença } \\
\text { renal crônica. }\end{array}$ \\
\hline $\begin{array}{l}\text { Importância da terapia nutricional } \\
\text { com ênfase nocálcio, fósforo e } \\
\text { potássio no tratamento da doença } \\
\text { renal crônica. }\end{array}$ & TELLES C, et al & 2015 & $\begin{array}{c}\text { Compreender através de uma análise } \\
\text { bibliográfica a importância da terapia } \\
\text { nutricional nos pacientes com doença renal } \\
\text { crônica, dando ênfase no potássio,cálcio e } \\
\text { fósforo. }\end{array}$ \\
\hline
\end{tabular}

Fonte: os autores. 
De acordo com Plácido et al (2021), os pacientes portadores de DRC são extremamente vulneráveis, necessitando de um acompanhamento frequente para avaliação do estado nutricional, visando minimizar os sintomas, as morbidades e mortalidades da patologia. Telles et al (2015), relata que a terapia nutricional apresenta um importante papel no tratamento dos pacientes portadores da doença renal crônica, contudo, esses pacientes costumam enfrentar algumas dificuldades para seguir essas orientações, uma vez que a sua dieta precisa conter a quantidade ideal de proteínas, calorias e paralelamente há a necessidade de regular alguns nutrientes como o fósforo, cálcio e potássio.

Pereira et al. (202I), enfatiza a importância dos ajustes na ingestão dos nutrientes como proteína, fósforo, cálcio, sódio e potássio a fim de garantir uma melhor qualidade de vida desses pacientes. Salientando os benefícios da adesão de um padrão alimentar saudável ligado a melhores resultados para o tratamento ou até mesmo na prevenção da DRC.

Banerjee T. et al. (2016) relata diversos padrões dietéticos, incluindo condutas com benefícios que visam melhoria na saúde desses pacientes com DRC. Também é citado que os padrões dietéticos com baixa carga de ácido na dieta podem retardar a progressão da DRC. Dietas à base de vegetais e frutas com baixa carga de ácidos e líquidos apresentam resultados satisfatórios nesses pacientes. Com relação à ingestão hídrica, os pacientes com DRC precisam restringir a ingestão de líquidos, visando assim prevenir a sobrecarga no aparelho renal (Lambert et al., 2017).

Perusso et al. (2019), descreve que o tratamento nutricional pode variar de acordo com a fase em que o paciente se encontra, assim a intervenção dietética poderá reduzir o risco de morte desses pacientes. Lambert K. et al. (2017) relata neste estudo, que diversos fatores podem gerar implicações sobre a adesão à dieta desses pacientes, mas estratégias podem ser empregues visando uma melhor aceitação da terapia nutricional.

\section{CONCLUSÃO}

Os resultados demonstram que os pacientes com doença renal crônica (DRC) são extremamente vulneráveis, com isso, ressalta-se a importância do acompanhamento desses pacientes com uma equipe multidisciplinar, principalmente com Nutricionistas, visando promover uma melhor adequação na dietoterapia desses pacientes. Uma dieta equilibrada 
possui um papel expressivo no tratamento de pacientes renais crônicos, sobretudo no que se refere a prevenir as complicações clínicas.

Salientamos a relevância da terapia nutricional adequada, visando melhoria na manutenção e recuperação do estado nutricional do paciente, controlando a ingestão de líquidos, sódio, potássio e proteínas, impedindo os edemas causados pelo desequilíbrio eletrolítico, regulando a ingestão de cálcio, fósforo e vitaminas promovendo assim uma melhor qualidade de vida desses pacientes. A intervenção dietética deve ser individual e adaptada de acordo ao estágio da patologia, sabe-se que na maioria das vezes é difícil a aceitação e a adesão dessa dieta pelos pacientes, por isso salienta-se a importância da orientação dos profissionais de saúde junto aos familiares e pacientes.

\section{REFERÊNCIAS}

AKIZAWA, T., OKUMURA, H., ALEXANDRE, A. FUKUSHIMA, A., KIYABU, G., $\&$

DOREY, J. Burden of Anemia in Chronic Kidney Disease Patients in Japan: A Literature Review. Therapeutic Apheresis and Dialysis, 22(5), 444-456, 2018.

AQUINO, R. D. C. D.; PHILIPPI, S. T. Nutrição Clínica: Estudos de Casos Comentados. São Paulo, SP: Editora Manole, 2016.

BANERJEE, T., LIU, Y., \& CREWS, D. C. (2016). Dietary Patterns and CKD Progression. Blood Purif. San Francisco. I7 (I), II7-II2. https://doi.org/ro.1159/ooo441072

BORGES, MICHELLE YASMINE. Consumo alimentar e fatores associados de pacientes com doença renal crônica em hemodiálise. 2019. $68 \mathrm{f}$. Trabalho de Conclusão de Curso (Graduação em Nutrição) - Universidade Federal de Mato Grosso, Faculdade de Nutrição, Cuiabá, 2019. Acesso em: 13 de Set. 2021.

CASTRO, M. C. M.Tratamento conservador de paciente com doença renal crônica que renuncia à diálise. Braz. J. Nephrol., v. 4I, n.I, p. 95-I02, mar. 2018. Acesso: Io de Set. 2021.

CHA RH, LEE H, LEE JP, KIM YS, KIM SG. The influence of blood pressure patterns on renal outcomes in patients with chronic kidney disease: The long-term follow up result of the APrODiTe-2 study. Medicine (Baltimore). 2020;99(8):ergzo9. doi:10.1097/MD.00ooooooooor9209

CHIBA, Y., MIZOGUCHI, I., HASEGAWA, H., OHASHI, M., ORII, N., NAGAI, T. \& 
YOSHIMOTO, T. Regulation of myelopoiesis by proinflammatory cytokines in infectious diseases. Cellular and molecular life sciences, 75(8), 1363-1376, 2018.

CUPPARI, L. Nutrição clínica no adulto. $4^{\circ}$ Edição. São Paulo, SP: Editora Manole, 2019.

FUKUCHIMA, RLM.; MENEZES A.L.C.; INOUYE K.; PAVARINI S.C.I.; ORLANDI

F.S. Fatores associados à qualidade de vida de pacientes renais crônicos em hemodiálise. Acta Paul Enferm; 2016. 29:518-24.

HILL NR, FATOBA ST, OKE JL, HIRST JA, O'CALLAGHAN CA, LASSERSON DS,

et al. Global Prevalence of Chronic Kidney Disease - A Systematic Review and MetaAnalysis. PLoS One. 2016; II(7):eors8765.

HONDA, H. et al. Iron Metabolism in Chronic Kidney Disease Patients.

Contributions to nephrology, v. 198, p. I03-III, 2019.

KIDNEY INTERNATIONAL (2020). KDIGO 2020 Clinical Practice Guideline for Diabetes Management in Chronic Kidney Disease. 98 (4), I-I2O. https://doi.org/ro.1016/j.kint.2020.06.o19.

LAMBERT $\mathrm{K}$, et al. An integrative review of the methodology and findings regarding dietary adherence in end stage kidney disease. BMC Nephrology, 2017; http://doi:10.1186/si2882-017-0734-z

LIMA, G. S. B. Aspectos nutricionais em doentes renais crônicos em tratamento conservador: uma revisão integrativa. Revista Eletrônica Acervo Científico, vol. 2I, p. e666o, 27 fev. 2021. Acesso em: ro de Set. 2021.

LUDVIG, T. C., et al. Avaliação do consumo de fósforo, potássio e alimentos processados e ultraprocessados em pacientes com doença renal crônica. Arch. Health Sci. (Online); 26(2).

Disponível: http://www.cienciasdasaude.famerp.br/index.php/racs/article/view/r459, abri-set.2019. Acesso em: II de Set. 2021.

MARTINS, M. D. A.; CARRILHO, F. J.; ALVES, V.A. F.; CASTILHO, E. Clínica Médica, Volume 3: Doenças Hematológicas, Oncologia, Doenças Renais. São Paulo, SP: Editora Manole, 2016.

MESTRINHO, B. V. ; LEITE, I. C. R. Doutor, tem uma pedra no meu rim?: perguntas e respostas sobre litíase urinária. São Paulo, SP: Editora Manole, 2019. 9788520461624. Acesso em: I2 de Set. 2021.

NEVES, P. D. M. M., et al. Censo Brasileiro de Diálise: análise de dados da década 2009-2018. São Paulo, SP. Disponível em: 
https://www.scielo.br/j/jbn/a/Dbk8RkskFYCSZGJv3FPpxWC/?format=pdf\&lang=pt Acesso em: io de Set. 2021.

NUHU, F., \& BHANDARI, S. Oxidative Stress and Cardiovascular Complications in Chronic Kidney Disease, the Impact of Anaemia. Pharmaceuticals, II(4), I03, 2018.

PAZ J. S; TAMASIA, G. A. Importância do autocuidado para paciente renal em tratamento conservador. [artigo] Registro: Faculdades Integradas do Vale do Ribeira, 2016.

PEREIRA, R. A. et al. Diet in Chronic Kidney Disease: an integrated approach to nutritional therapy. Revista da Associação Médica Brasileira [online]. 2020, v. 66, n. I. Acesso em: I6 de Set. 2021.

PERUSSO, F. K. G, et al. Alimentação e hábitos de vida na doença renal crônica. Revista Caderno de Medicina, 2019; 2(2). Acesso em II de Set. 202I.

PLÁCIDO, E. DA S.; LIMA, W. L. , et al. Terapia nutricional em pacientes com Doença Renal Crônica: Revisão narrativa. Research, Society and Development, [S.1.], v.io, n.4, p.e8II04I37II, 2021. DOI:Io.33448/rsd-vioi4.I37II. Disponível em: https://rsdjournal.org/index.php/rsd/article/view/137II. Acesso em: I3 de Set. 202I.

RIBEIRO, WA; JORGE, BO; QUEIROZ, RS. Repercussões da hemodiálise no paciente com doença renal crônica: uma revisão da literatura. Revista

Pró-UniverSUS. 2020 Jan./Jun.;II (I): 88-97

RODRIGUES, I. D. Relação entre consumo alimentar e ganho de peso interdialítico em doentes renais crônicos. Revista saúde.com, [s. 1.], v. 13, n.I, p.779-785,2017. disponível em: https://periodicos2.uesb.br/index.php/rsc/article/view/445. Acesso em: II de Set. 202I.

ROSS, A. C.; CABALLERO, B.; COUSINS, R. J.; TUCKER, K. L.; ZIEGLER, T. R. Nutrição Moderna de Shils na Saúde e na Doença. Barueri, SP: Editora Manole, 2016.

SILVA, A. S. C. DA; VAZ, E. M. Contribuição da dieta e água alcalina na redução de complicações da doença renal crônica. Revista da Associação Brasileira de Nutrição RASBRAN, v. II, n. I, p. I76-191, 2020. DOI:

I0.47320/rasbran.2020.1772. Disponível em: https://www.rasbran.com.br/rasbran/article/view/r772. Acesso em II de Set. 202I.

SILVA, J. S. Consumo de alimentos ricos em fósforo nos pacientes renais crônicos em hemodiálise. Trabalho de conclusão de curso - Universidade Federal de Pernambuco. Vitória de Santo Antão, 2016. Acesso em: I8 de Set. 2021. 
STOMPÓR T, PERKOWSKA-PTASINSKA A. Hypertensive kidney disease: a true epidemic or rare disease?. Pol Arch Intern Med. 2020 Feb 27;130(2):130-139. doi: I0.20452/pamw.15150. Epub 2020 Jan 22.

TELLES, C.; BOITA, E. R. F. Importância da terapia nutricional com ênfase no cálcio, fósforo e potássio no tratamento da doença renal crônica. Perspectiva, Erechim, v. 39, n.145, p. I43-I54, março/2015. disponível em: https://www.uricer.edu.br/site/pdfs/perspectiva/I45_489.pdf. Acesso em: II de Set. 202I.

VERHELST D. Characteristics and epidemiology of chronic kidney disease. Soins. 2018; $63(826): 14-16$. 